\title{
Serum Albumin and Disease Severity of Non-Cystic Fibrosis Bronchiectasis
}

\author{
Seung Jun Lee MD, Hyo-Jung Kim MD, Ju-Young Kim MD, Sunmi Ju MD, Sujin Lim MD, \\ Jung Wan Yoo MD, Sung-Jin Nam MD, Gi Dong Lee MD, Hyun Seop Cho MD, \\ Rock Bum Kim MD, Yu Ji Cho MD, Yi Yeong Jeong MD, Ho Cheol Kim MD, and \\ Jong Deog Lee MD
}

\begin{abstract}
BACKGROUND: A clinical classification system has been developed to define the severity and predict the prognosis of subjects with non-cystic fibrosis (CF) bronchiectasis. We aimed to identify laboratory parameters that are correlated with the bronchiectasis severity index (BSI) and FACED score. METHODS: The medical records of 107 subjects with non-CF bronchiectasis for whom BSI and FACED scores could be calculated were retrospectively reviewed. The correlations between the laboratory parameters and BSI or FACED score were assessed, and multiple-linear regression analysis was performed to identify variables independently associated with BSI and FACED score. An additional subgroup analysis was performed according to sex. RESULTS: Among all of the enrolled subjects, $49(45.8 \%)$ were male and $58(54.2 \%)$ were female. The mean BSI and FACED scores were $9.43 \pm 3.81$ and $1.92 \pm 1.59$, respectively. The serum albumin level $(r=-0.49)$, bilirubin level $(r=-0.31)$, C-reactive protein level $(r=0.22)$, hemoglobin level $(r=-0.2)$, and platelet/lymphocyte ratio $(r=0.31)$ were significantly correlated with BSI. Meanwhile, serum albumin $(r=-0.37)$ and bilirubin level $(r=-0.25)$ showed a significant correlation with the FACED score. Multiple-linear regression analysis showed that the serum bilirubin level was independently associated with BSI, and the serum albumin level was independently associated with both scoring systems. Subgroup analysis revealed that the level of uric acid was also a significant variable independently associated with the BSI in male bronchiectasis subjects. CONCLUSIONS: Several laboratory variables were identified as possible prognostic factors for non-CF bronchiectasis. Among them, the serum albumin level exhibited the strongest correlation and was identified as an independent variable associated with the BSI and FACED scores. Key words: non-cystic fibrosis bronchiectasis; serum albumin; bronchiectasis severity index; FACED score. [Respir Care 2017;62(8):1075-1084. (C) 2017 Daedalus Enterprises]
\end{abstract}

\section{Introduction}

Bronchiectasis, a chronic respiratory disease defined as a permanent dilatation of the bronchi due to recurrent inflam-

Drs Seung Jun Lee, Hyo-Jung Kim, Ju-Young Kim, Ju, Lim, Yoo, Cho, Jeong, and Jong Deog Lee are affiliated with the Division of Pulmonology and Allergy; Drs Nam, Gi Dong Lee, and Ho Cheol Kim are affiliated with the Division of Pulmonology and Allergy; and Dr Cho is affiliated with the Division of Nephrology, Department of Internal Medicine, and Dr Rock Bum Kim is affiliated with the Department of Preventive Medicine and Environmental Health Center, Gyeongsang National University Hospital, Gyeongsang National University School of Medicine, Jinju, Republic of Korea. mation and infection of the airways, causes agonizing symptoms, including chronic cough, sputum production, and hemoptysis. ${ }^{1}$ Cystic fibrosis is the most common inheritable cause of bronchiectasis in western populations, and most bron-

\footnotetext{
The authors have disclosed no conflicts of interest.

Correspondence: Jong Deog Lee PhD MD, Division of Pulmonology and Allergy, Department of Internal Medicine, School of Medicine, Gyeongsang National University, 92 Chilam-dong, Jinju, Gyeongnam 660751, Republic of Korea. E-mail: 1jd8611@nate.com.
}

DOI: $10.4187 /$ respcare.05276 
chiectasis studies have focused on cystic fibrosis. Non-cystic fibrosis bronchiectasis (non-CF bronchiectasis) was regarded as an orphan disease for decades. However, there is a growing interest in, and unmet need for, non-CF bronchiectasis studies because the pathophysiology, treatment, and prognosis of non-CF bronchiectasis may be different from those of cystic fibrosis. ${ }^{2}$ Data on the management of non-CF bronchiectasis are lacking, and existing guidelines are based on cystic fibrosis studies and expert opinion. ${ }^{3}$

Systematic assessment of disease severity is fundamental to understand the natural course of disease and will be helpful to predict the prognosis of subjects with non-CF bronchiectasis. The bronchiectasis severity index (BSI) and FACED instruments have been developed to assess the severity of non-CF bronchiectasis. ${ }^{4,5}$ The FACED score encompasses 5 variables: Pseudomonas colonization, dyspnea scale, $\mathrm{FEV}_{1}$, age, and the number of affected lobes. The BSI includes 9 variables, including the 5 variables of the FACED score with the addition of body mass index, hospital admission, exacerbation, and colonization with other organisms. Both FACED and BSI scores are valid for predicting the mortality of patients with non-CF bronchiectasis. However, it is not always feasible to calculate these clinical scores in real practice.

Several biomarkers have been developed for the diagnosis, evaluation of treatment response, and prediction of prognosis of airway diseases. COPD and asthma are representative diseases for which biomarker studies were extensively performed for decades. ${ }^{6,7}$ The eosinophil counts in induced sputum can effectively guide the dose of medication, ${ }^{8}$ and exhaled nitric oxide can predict eosinophilic inflammation and steroid responsiveness. ${ }^{9}$ Meanwhile, the fibrinogen level is a well-established blood biomarker in patients with COPD, predicting mortality and the risk of exacerbation. ${ }^{10}$ Although proteomic and metabolomic biomarkers are under investigation, ${ }^{11,12}$ further studies are needed to establish their clinical implications in airway diseases. Regarding biomarkers in patients with non-CF bronchiectasis, scarce data exist to the best of our knowledge. Thus, the aim of the current study was to discover blood biomarkers that may be useful to predict the prognosis of subjects with non-CF bronchiectasis using the BSI and FACED scores.

\section{Methods}

\section{Study Population}

The study group included 107 subjects with non-CF bronchiectasis who visited Gyeongsang National University Hospital (Jinju, Korea) from July 2013 to August 2014. The diagnosis of bronchiectasis was made by highresolution computed tomography in subjects with a compatible clinical history. The morphologic criteria of highresolution computed tomography include (1) a larger size

\section{QUICK LOOK}

\section{Current knowledge}

The bronchiectasis severity index and FACED score are multidimensional scoring systems that were developed to define the severity of non-CF bronchiectasis. Both scores accurately predicted exacerbation and mortality of patients with non-CF bronchiectasis. However, the association between laboratory variables and scoring systems was not validated.

\section{What this paper contributes to our knowledge}

In this retrospective study, several laboratory biomarkers, including serum albumin, bilirubin, hemoglobin, C-reactive protein, platelet/lymphocyte ratio, and uric acid, were significantly correlated with bronchiectasis severity index and/or FACED score. Among these parameters, serum albumin showed the strongest and independent association with both scoring systems.

of the bronchial internal diameter than the accompanying pulmonary artery and (2) a lack of tapering of the bronchi in the peripheral lungs. ${ }^{13}$ Clinical symptoms consistent with bronchiectasis include chronic cough, productive sputum, exertional dyspnea, and recurrent lower respiratory infection. $^{3}$ The exclusion criteria were as follows: (1) a diagnosis of bronchiectasis based solely on partial lung images of abdominal computed tomography; (2) inability to verify the results of a pulmonary function test; (3) the absence of sputum bacteriologic results; (4) traction bronchiectasis due to interstitial lung disease or other pulmonary diseases; (5) non-tuberculous mycobacterial lung disease; and (6) coexistence of an active malignant disease. The diagnosis of non-tuberculous mycobacterial lung disease was made according to the official statement of the American Thoracic Society and the Infectious Disease Society of America guidelines. ${ }^{14}$ This study was approved by the institutional review board of Gyeongsang National University Hospital (approval GNUH2016-02-005).

\section{Data Collection and Study Design}

All of the data were collected from a retrospective review of the subjects' medical records. Data on baseline demographic characteristics and clinical variables, obtained to calculate the BSI and FACED scores, included age, body mass index, $\mathrm{FEV}_{1}$, Medical Research Council dyspnea score, modified Medical Research Council dyspnea score, radiologic appearance of dilated bronchi (cylindrical vs varicose vs cystic), number of affected lobes, Pseudomonas colonization, colonization with other organisms, 
number of hospital admissions, and number of exacerbations. Comorbid diseases and laboratory values, including the white blood cell count, neutrophil/lymphocyte ratio, platelet count, platelet/lymphocyte ratio, and hematocrit, as well as the serum levels of hemoglobin, protein, albumin, total bilirubin, uric acid, and C-reactive protein, were also obtained. Spirometry was performed according to the guidelines of the American Thoracic Society and European Respiratory Society. ${ }^{15}$ COPD was diagnosed by spirometry if the post-bronchodilator $\mathrm{FEV}_{1} / \mathrm{FVC}$ was $<0.7$ according to the Global Initiative for Chronic Obstructive Lung Disease guidelines. Peripheral blood samples were collected, and spirometry was conducted when the subjects' condition was considered clinically stable at the outpatient department during their regular follow-up. Radiologic assessments of bronchiectasis were performed using the modified Reiff score. ${ }^{16}$ The definition of chronic colonization was the isolation of bacteria in sputum $\geq 2$ times, $\geq 3$ months apart within 1 y. ${ }^{5}$ Exacerbations were defined as worsening symptoms, such as increasing sputum volume or purulence, worsening dyspnea, increased cough, or increased fatigue/malaise. ${ }^{3}$

Based on verified medical records, the BSI and FACED scores were calculated in enrolled subjects with non-CF bronchiectasis. Laboratory values that were significantly correlated with subjects' BSI and FACED scores were analyzed. An additional subgroup analysis was performed according to sex.

\section{Statistical Analysis}

Unpaired $t$ test and one-way analysis of variance were used if the distribution was normal, or Mann-Whitney U test and Kruskal-Wallis test were used if the distribution was not normal to compare categorical variables. The correlation coefficients and significance of the association between 2 continuous variables were analyzed by Pearson's correlation test when both variables were normally distributed. Spearman's rho correlation test was used when one or both of the continuous variables were not normally distributed. Multiple linear regression analysis was performed to identify variables independently associated with the dependent variables (ie, the BSI and FACED scores). Only statistically significant variables in the correlation test were entered into the multivariate analysis. A $P$ value $<.05$ was considered to indicate statistical significance. Statistical analyses were performed using SPSS 21.0 for Windows (SPSS, Chicago, Illinois).

\section{Results}

\section{Baseline Characteristics}

In total, 283 patients with non-CF bronchiectasis were identified initially, of whom 226 underwent chest com-

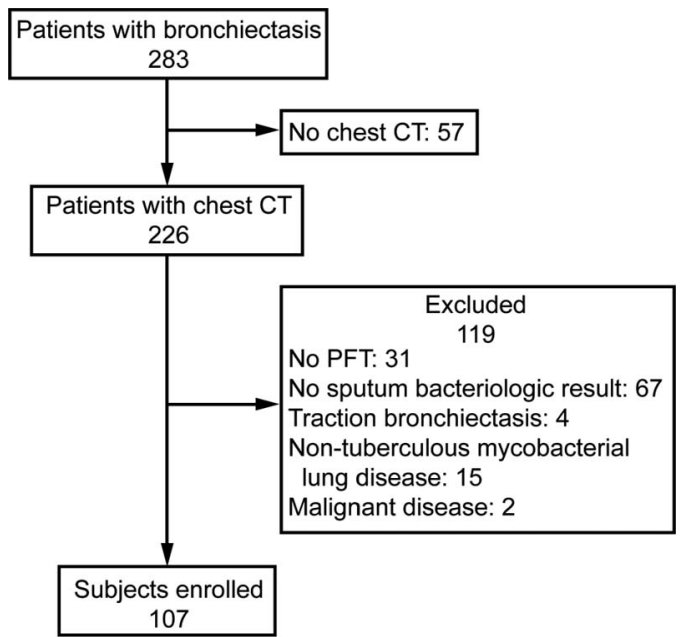

Fig. 1. Flow chart. CT = computed tomography, PFT = pulmonary function testing.

puted tomography. An additional 119 patients were excluded for the reasons outlined in Figure 1. Finally, 107 subjects were enrolled for the final analysis. The mean age of the enrolled subjects was $62.5 \pm 10.1 \mathrm{y}$, and 58 subjects $(54.2 \%)$ were female. The mean $\mathrm{FEV}_{1}$ was $1.78 \pm 0.68 \mathrm{~L}$, and the mean percent-of-predicted value of $\mathrm{FEV}_{1}$ was $67.8 \pm 23.0$. Most subjects $(71 \%)$ were never-smokers. A history of hospital admissions and exacerbation was present in 71 subjects $(66.4 \%)$ and 78 subjects $(72.9 \%)$, respectively. There were 24 subjects $(22.4 \%)$ with Pseudomonas colonization, and 13 subjects (12.1\%) were colonized with other organisms. Cylindrical bronchiectasis was the most common pattern on high-resolution computed tomography, followed by a cystic and varicose bronchiectasis pattern. The mean duration of disease in subjects with non-CF bronchiectasis was $56.9 \pm 37.7$ months. The mean BSI and FACED scores of the enrolled subjects were $9.43 \pm 3.81$ and $1.92 \pm 1.59$, respectively. The distribution of subjects according to the tertiles of the BSI and FACED scores is shown in Figure 2. The ranges of scores for the low, intermediate, and high group according to the BSI were $0-4,5-8$, and $\geq 9$ points, and those according to the FACED score were $0-2,3-4$, and 5-7 points, respectively. ${ }^{4,5}$. Subjects with a low FACED score predominated, whereas the high-score group predominated according to BSI classification. COPD was present in 50.5\% of subjects with non-CF bronchiectasis. Other common comorbid diseases included hypertension, diabetes, and asthma (Table 1). A comparison of the characteristics of the male and female subjects is provided in Table 1. Neversmokers were more common, the number of exacerbations in the previous year was lower, and COPD was less commonly present in female subjects than in male subjects with non-CF bronchiectasis. Table 2 lists the laboratory values of the enrolled subjects. 


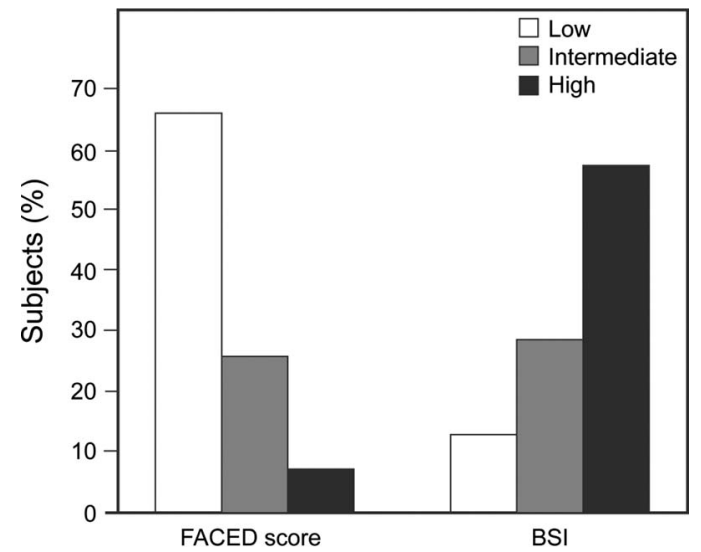

Fig. 2. Subject distribution according to FACED score and bronchiectasis severity index (BSI).

\section{Factors Correlated With BSI and FACED Score}

Among the various laboratory values, 5 factors, including the serum albumin level, bilirubin level, hemoglobin level, C-reactive protein level, and platelet/lymphocyte ratio, were significantly correlated with the BSI. Factors significantly correlated with the FACED score were the serum albumin and bilirubin level (Table 3). The serum albumin and bilirubin levels significantly correlated with both the BSI and FACED scores. The correlation coefficients of the serum albumin were higher than those for the other variables. Multiple-linear regression analysis identified variables independently associated with the BSI and FACED scores. The serum albumin and bilirubin levels were independent variables associated with the BSI score. The serum albumin level was the only variable independently associated with the FACED score (Table 4). The serum albumin level was significantly different among subjects with mild, intermediate, and severe BSI scores. The association between serum bilirubin level and BSI was weak and statistically insignificant. There were not significant differences for FACED score in the serum albumin and bilirubin level across severity groups (Fig. 3). Additional subgroup analysis according to sex showed that uric acid was significantly correlated and independently associated with the BSI in male subjects with non-CF bronchiectasis (Tables 3 and 5).

\section{Discussion}

The current study aimed to identify laboratory variables that show a significant correlation with the BSI and FACED scores of subjects with non-CF bronchiectasis; in other words, our main objective was to indirectly uncover possible prognostic factors using well-validated prognostic scoring systems. The serum albumin level, bilirubin level, C-reactive protein level, platelet/lympho- cyte ratio, and hemoglobin level showed significant correlations with the BSI and/or FACED score, implying that these laboratory variables could be used as prognostic factors. The serum albumin level showed a significant correlation with both scoring systems, and the degree of correlation was higher than that for the other factors. After multivariate analysis, the serum albumin level achieved a significant independent association with the BSI and FACED scores.

We enrolled 107 subjects with non-CF bronchiectasis who visited a single tertiary referral hospital in South Korea. The demographic and clinical characteristics of our subjects showed many similarities with those of subjects from other studies. In many previous reports, non-CF bronchiectasis occurred more commonly in females than in males..$^{4,17-19}$ Morrissey and Harper ${ }^{20}$ reported that non-CF bronchiectasis is more common and virulent in females because of different immune responses. Correspondingly, slightly more female subjects were enrolled in this study. Female subjects showed relatively higher lung function, a lower number of exacerbations, and lower uric acid and C-reactive protein levels compared with the male subjects in this study. Loebinger et al, ${ }^{21}$ meanwhile, reported that male sex was an independent predictor of long-term mortality in subjects with bronchiectasis. The prognostic difference between male and female subjects needs to be validated in the future.

The current study showed that chronic colonization with Pseudomonas was present in $22.4 \%$ of the enrolled subjects. The frequency of Pseudomonas isolation in subjects with non-CF bronchiectasis is $12-31 \%$ according to a review article by King et al. ${ }^{22}$ They reported that the colonization of Haemophilus influenza is as common as that of Pseudomonas. However, colonization with Haemophilus was uncommon in this study (data not shown).

An overlap between COPD and bronchiectasis has been highlighted because the 2 diseases coexist frequently, and patients with both diseases show increased mortality. ${ }^{23}$ Bronchiectasis and COPD have common clinical features, including cough, sputum, and exacerbation. However, the pathogenesis, diagnostic tools, and treatment modality are somewhat different. Over half of the subjects with non-CF bronchiectasis had spirometry-diagnosed COPD as a comorbid disease in this study. Because $71.0 \%$ of the subjects were never-smokers, the interaction between bronchiectasis and COPD may be more vital. Previous studies ${ }^{24,25}$ proved that bronchiectasis increased the mortality of subjects with COPD and vice versa. More studies are needed to assess the impact of COPD on the prognosis of subjects with bronchiectasis.

The BSI and FACED scoring systems were developed by 2 different research groups. We found a different pattern in terms of disease severity classification between the BSI and FACED instruments; $66.4 \%$ of our subjects were 
Table 1. Baseline Characteristics of the Study Subjects With Non-Cystic Fibrosis Bronchiectasis

\begin{tabular}{|c|c|c|c|c|}
\hline Characteristics & All Subjects $(N=107)$ & Male $(n=49)$ & Female $(n=58)$ & $P^{*}$ \\
\hline Age, mean \pm SD y & $62.5 \pm 10.1$ & $62.1 \pm 10.3$ & $62.9 \pm 10.1$ & .66 \\
\hline Body mass index, mean $\pm \mathrm{SD} \mathrm{kg} / \mathrm{m}^{2}$ & $21.7 \pm 3.40$ & $22.0 \pm 3.87$ & $21.5 \pm 2.97$ & .38 \\
\hline $\mathrm{FEV}_{1} / \mathrm{FVC}$, mean $\pm \mathrm{SD}$ & $68.5 \pm 12.8$ & $66.0 \pm 12.9$ & $70.6 \pm 12.5$ & .06 \\
\hline $\mathrm{FEV}_{1}$, mean $\pm \mathrm{SD} \mathrm{L}$ & $1.78 \pm 0.68$ & $1.96 \pm 0.81$ & $1.63 \pm 0.52$ & .02 \\
\hline $\mathrm{FEV}_{1}$, mean $\pm \mathrm{SD} \%$ predicted & $67.8 \pm 23.0$ & $63.1 \pm 24.2$ & $71.7 \pm 21.4$ & .052 \\
\hline Smoking status, $n(\%)$ & & & & $<.001$ \\
\hline Current smoker & $7(6.5)$ & $6(12.2)$ & $1(1.7)$ & \\
\hline Former smoker & $24(22.4)$ & $23(46.9)$ & $1(1.7)$ & \\
\hline Never-smoker & $76(71.0)$ & $20(40.8)$ & $56(96.6)$ & \\
\hline History of respiratory hospitalization, $n(\%)$ & & & & .83 \\
\hline Yes & $71(66.4)$ & $32(65.3)$ & $39(67.2)$ & \\
\hline No & $36(33.6)$ & $17(34.7)$ & $19(32.8)$ & \\
\hline History of exacerbation, $n(\%)$ & & & & .32 \\
\hline Yes & $78(72.9)$ & $38(77.6)$ & $40(69.0)$ & \\
\hline No & $29(27.1)$ & $11(22.4)$ & $18(31.0)$ & \\
\hline Number of exacerbations in previous $y$, mean $\pm \mathrm{SD}$ & $1.46 \pm 1.48$ & $1.98 \pm 1.81$ & $1.02 \pm 0.93$ & .001 \\
\hline Chronic colonization with Pseudomonas, $n(\%)$ & & & & .99 \\
\hline Yes & $24(22.4)$ & $11(22.4)$ & $13(22.4)$ & \\
\hline No & $83(77.6)$ & $38(77.6)$ & $45(77.6)$ & \\
\hline Chronic colonization with other organism, $n(\%)$ & & & & .07 \\
\hline Yes & $13(12.1)$ & $9(18.4)$ & $4(6.9)$ & \\
\hline No & $94(87.9)$ & $40(81.6)$ & $54(93.1)$ & \\
\hline Number of affected lobes, mean $\pm \mathrm{SD}$ & $3.24 \pm 1.52$ & $3.39 \pm 1.46$ & $3.12 \pm 1.58$ & .37 \\
\hline Pattern of bronchiectasis, $n(\%)$ & & & & .46 \\
\hline Cylindrical & $68(63.6)$ & $34(69.4)$ & $34(58.6)$ & \\
\hline Varicose & $4(3.7)$ & $2(4.1)$ & $2(3.4)$ & \\
\hline Cystic & $35(32.7)$ & $13(26.5)$ & $22(37.9)$ & \\
\hline FACED score, mean $\pm \mathrm{SD}$ & $1.92 \pm 1.59$ & $2.18 \pm 1.54$ & $1.69 \pm 1.61$ & .11 \\
\hline $\mathrm{BSI}$, mean $\pm \mathrm{SD}$ & $9.43 \pm 3.81$ & $10.1 \pm 4.09$ & $8.90 \pm 3.50$ & .12 \\
\hline \multicolumn{5}{|l|}{ Comorbid diseases, $n(\%)$} \\
\hline COPD & $54(50.5)$ & $30(61.2 \%)$ & $24(41.4)$ & .041 \\
\hline Hypertension & $27(25.2)$ & $11(22.4)$ & $16(27.6)$ & .54 \\
\hline Diabetes & $19(17.8)$ & $9(18.4)$ & $10(17.2)$ & .88 \\
\hline Cardiovascular disease & $5(4.7)$ & $3(6.1)$ & $2(3.4)$ & .51 \\
\hline Bronchial asthma & $24(22.4)$ & $11(22.4)$ & $13(22.4)$ & .99 \\
\hline Previous history of tuberculosis & $28(26.2)$ & $15(30.6)$ & $13(22.4)$ & .34 \\
\hline Depression & $6(5.6)$ & $1(2.0)$ & $5(8.6)$ & .14 \\
\hline
\end{tabular}

in the low FACED score group, whereas the low BSI group included only $13.1 \%$ of the subjects. The classifications of the systems were found to be comparable in a report of Minov et al, ${ }^{26}$ which showed that 43.2 and $45.9 \%$ of subjects belonged to the low-BSI and low-FACED score groups, respectively. However, larger multi-center European cohorts showed that the subjects were predominantly classified as a moderate-to-severe group based on BSI classification, and a mild bronchiectasis group predominated according to the BSI classification. ${ }^{27}$ Brill et $\mathrm{al}^{17}$ reported that $28 \%$ of subjects with non-CF bronchiectasis were classified into the low BSI group, but they did not discuss the FACED score in their study. Although both scoring systems accurately predict the mortality of subjects with non-CF bronchiectasis, ${ }^{4,5}$ a discrepancy could exist in terms of severity classification; however, the clinical importance of this phenomenon remains unclear.

The importance of systemic inflammation and consequent systemic comorbidities are well-established in patients with COPD. ${ }^{28}$ To date, the evidence of systemic inflammation in patients with non-CF bronchiectasis is insufficient. However, a few studies have suggested that systemic inflammatory responses are increased in stable bronchiectasis subjects. The plasma concentration of 
Table 2. Laboratory Findings of the Study Subjects

\begin{tabular}{|c|c|c|c|c|}
\hline Values & All Subjects $(N=107)$ & Male $(n=49)$ & Female $(n=58)$ & $P^{*}$ \\
\hline White blood cell, $10^{9} / \mathrm{L}$ & $7.11 \pm 2.34$ & $7.62 \pm 2.55$ & $6.68 \pm 2.07$ & .038 \\
\hline Neutrophil, \% & $59.3 \pm 11.4$ & $60.4 \pm 10.9$ & $58.4 \pm 11.9$ & .37 \\
\hline Lymphocyte, \% & $30.6 \pm 10.3$ & $28.8 \pm 9.64$ & $32.1 \pm 10.6$ & .10 \\
\hline NLR & $2.54 \pm 2.55$ & $2.60 \pm 1.84$ & $2.49 \pm 3.05$ & .82 \\
\hline Platelet, $10^{9} / \mathrm{L}$ & $255.9 \pm 80.6$ & $252.6 \pm 83.3$ & $258.7 \pm 78.8$ & .70 \\
\hline PLR & $138.0 \pm 66.0$ & $132.9 \pm 52.1$ & $142.4 \pm 76.0$ & .46 \\
\hline Hemoglobin, g/dL & $12.6 \pm 1.62$ & $13.2 \pm 1.75$ & $12.2 \pm 1.34$ & .001 \\
\hline BUN, mg/dL & $14.1 \pm 7.55$ & $15.3 \pm 8.98$ & $13.1 \pm 5.99$ & .14 \\
\hline Creatinine, $\mathrm{mg} / \mathrm{dL}$ & $0.75 \pm 0.34$ & $0.91 \pm 0.43$ & $0.62 \pm 0.15$ & $<.001$ \\
\hline Uric acid, $\mathrm{mg} / \mathrm{dL}$ & $4.76 \pm 1.63$ & $5.64 \pm 1.75$ & $4.01 \pm 1.06$ & $<.001$ \\
\hline Total protein, g/dL & $7.13 \pm 0.57$ & $7.18 \pm 0.59$ & $7.09 \pm 0.56$ & .44 \\
\hline Albumin, g/dL & $4.11 \pm 0.52$ & $4.03 \pm 0.60$ & $4.18 \pm 0.45$ & .15 \\
\hline Total bilirubin, $\mathrm{mg} / \mathrm{dL}$ & $0.54 \pm 0.33$ & $0.60 \pm 0.38$ & $0.48 \pm 0.26$ & .052 \\
\hline C-reactive protein, $\mathrm{mg} / \mathrm{L}$ & $8.21 \pm 11.1$ & $11.3 \pm 13.1$ & $5.64 \pm 8.27$ & .01 \\
\hline \multicolumn{5}{|c|}{$\begin{array}{l}\text { Data are means } \pm \text { SD. } \\
* P \text { values represent differences between male and female subjects. } \\
\text { NLR }=\text { neutrophil/lymphocyte ratio } \\
\text { PLR }=\text { platelet/lymphocyte ratio } \\
\text { BUN = blood urea nitrogen }\end{array}$} \\
\hline
\end{tabular}

Table 3. Correlation Coefficient for Continuous Variables

\begin{tabular}{|c|c|c|c|c|c|c|c|c|c|c|c|c|}
\hline \multirow[t]{2}{*}{ Variables } & \multicolumn{2}{|c|}{ BSI, All Subjects } & \multicolumn{2}{|c|}{$\begin{array}{l}\text { FACED Score, } \\
\text { All Subjects }\end{array}$} & \multicolumn{2}{|c|}{$\begin{array}{l}\text { BSI, Male } \\
\text { Subjects }\end{array}$} & \multicolumn{2}{|c|}{$\begin{array}{l}\text { FACED Score, } \\
\text { Male Subjects }\end{array}$} & \multicolumn{2}{|c|}{$\begin{array}{l}\text { BSI, Female } \\
\text { Subjects }\end{array}$} & \multicolumn{2}{|c|}{$\begin{array}{c}\text { FACED } \\
\text { Score, Female } \\
\text { Subjects }\end{array}$} \\
\hline & $\mathrm{r}$ & $P$ & $\mathrm{r}$ & $P$ & $\mathrm{r}$ & $P$ & $\mathrm{r}$ & $P$ & $\mathrm{r}$ & $P$ & $\mathrm{r}$ & $P$ \\
\hline White blood cell & 0.06 & .55 & 0.16 & .10 & 0.16 & .28 & 0.13 & .37 & -0.13 & .34 & 0.16 & .23 \\
\hline Neutrophil & 0.09 & .36 & 0.10 & .32 & 0.31 & .033 & 0.30 & .038 & -0.13 & .34 & -0.10 & .46 \\
\hline Lymphocyte & -0.13 & .20 & -0.14 & .15 & -0.30 & .040 & -0.33 & .02 & 0.07 & .63 & 0.08 & .57 \\
\hline NLR & 0.08 & .43 & 0.12 & .22 & 0.26 & .07 & 0.31 & .033 & -0.14 & .31 & -0.09 & .51 \\
\hline Platelet & 0.19 & .053 & 0.15 & .13 & 0.23 & .11 & 0.22 & .13 & 0.15 & .25 & 0.08 & .53 \\
\hline PLR & 0.31 & .001 & 0.14 & .16 & 0.38 & .007 & 0.34 & .02 & 0.30 & .02 & -0.02 & .89 \\
\hline Hemoglobin & -0.20 & .036 & -0.10 & .33 & -0.29 & .046 & -0.33 & .02 & -0.25 & .059 & -0.06 & .66 \\
\hline BUN & -0.09 & .36 & -0.09 & .37 & -0.17 & .24 & -0.24 & .10 & -0.07 & .61 & -0.03 & .81 \\
\hline Creatinine & -0.12 & .22 & -0.03 & .77 & -0.39 & .008 & -0.37 & .009 & -0.11 & .41 & 0.02 & .90 \\
\hline Uric acid & -0.07 & .47 & 0.04 & .71 & -0.37 & .008 & -0.20 & .17 & 0.14 & .29 & 0.14 & .29 \\
\hline Total protein & -0.11 & .26 & -0.10 & .33 & -0.07 & .64 & -0.02 & .89 & -0.18 & .18 & -0.17 & .20 \\
\hline Albumin & -0.49 & $<.001$ & -0.37 & $<.001$ & -0.64 & $<.001$ & -0.57 & $<.001$ & -0.29 & .03 & -0.17 & .20 \\
\hline Total bilirubin & -0.31 & .001 & -0.25 & .01 & -0.41 & .004 & -0.39 & .006 & -0.30 & .02 & -0.23 & .08 \\
\hline $\mathrm{C}$-reactive protein & 0.22 & .02 & 0.17 & .09 & 0.23 & .12 & 0.28 & .050 & 0.18 & .18 & 0.04 & .80 \\
\hline $\begin{array}{l}\text { BSI = bronchiectasis sev } \\
\text { NLR = neutrophil/lymph } \\
\text { PLR = platelet/lymphoc } \\
\text { BUN = blood urea nitro }\end{array}$ & $\begin{array}{l}\text { index } \\
\text { ratio } \\
\text { io }\end{array}$ & & & & & & & & & & & \\
\hline
\end{tabular}

TNF- $\alpha$ was reported to be higher in non-CF bronchiectasis subjects than in healthy controls, and the level of TNF- $\alpha$ showed a significant correlation with the C-reactive protein level, erythrocyte sedimentation rate, and peripheral blood neutrophil count. ${ }^{29}$ The authors also showed that the concentration of TNF- $\alpha$ was associated with the disease severity of non-CF bronchiectasis, ${ }^{29}$ suggesting a possible prognostic role of systemic inflammatory biomarkers in non-CF bronchiectasis, similar to COPD. Gale et $\mathrm{al}^{30}$ reported that the plasma interleukin-6 level was elevated, and the albumin level was reduced, in subjects with non-CF bronchiectasis compared with those in control subjects, and Ergan Arsava et $\mathrm{al}^{31}$ reported that the C-reactive protein level, white blood cell count, and fibrinogen level 
Table 4. Multiple Linear Regression Analysis of the Laboratory Variables Associated With the Bronchiectasis Severity Index and FACED Scores

\begin{tabular}{|c|c|c|c|c|}
\hline Dependent Variables & Explanatory Variables & $\beta$ Coefficient & $95 \% \mathrm{CI}$ & $P$ \\
\hline \multirow[t]{5}{*}{ BSI } & PLR & 0.01 & -0.01 to 0.02 & .37 \\
\hline & Hemoglobin (g/dL) & 0.37 & -0.09 to 0.83 & .11 \\
\hline & Albumin $(\mathrm{g} / \mathrm{dL})$ & -3.78 & -5.23 to -2.33 & $<.001$ \\
\hline & Bilirubin (mg/dL) & -3.50 & -5.56 to -1.43 & .001 \\
\hline & C-reactive protein $(\mathrm{mg} / \mathrm{L})$ & 0.02 & -0.04 to 0.08 & .52 \\
\hline \multirow[t]{2}{*}{ FACED score } & Albumin $(\mathrm{g} / \mathrm{dL})$ & -1.18 & -1.72 to -0.65 & $<.001$ \\
\hline & Bilirubin (mg/dL) & -0.84 & -1.71 to 0.02 & .055 \\
\hline
\end{tabular}

BSI $=$ bronchiectasis severity index PLR $=$ platelet/lymphocyte ratio
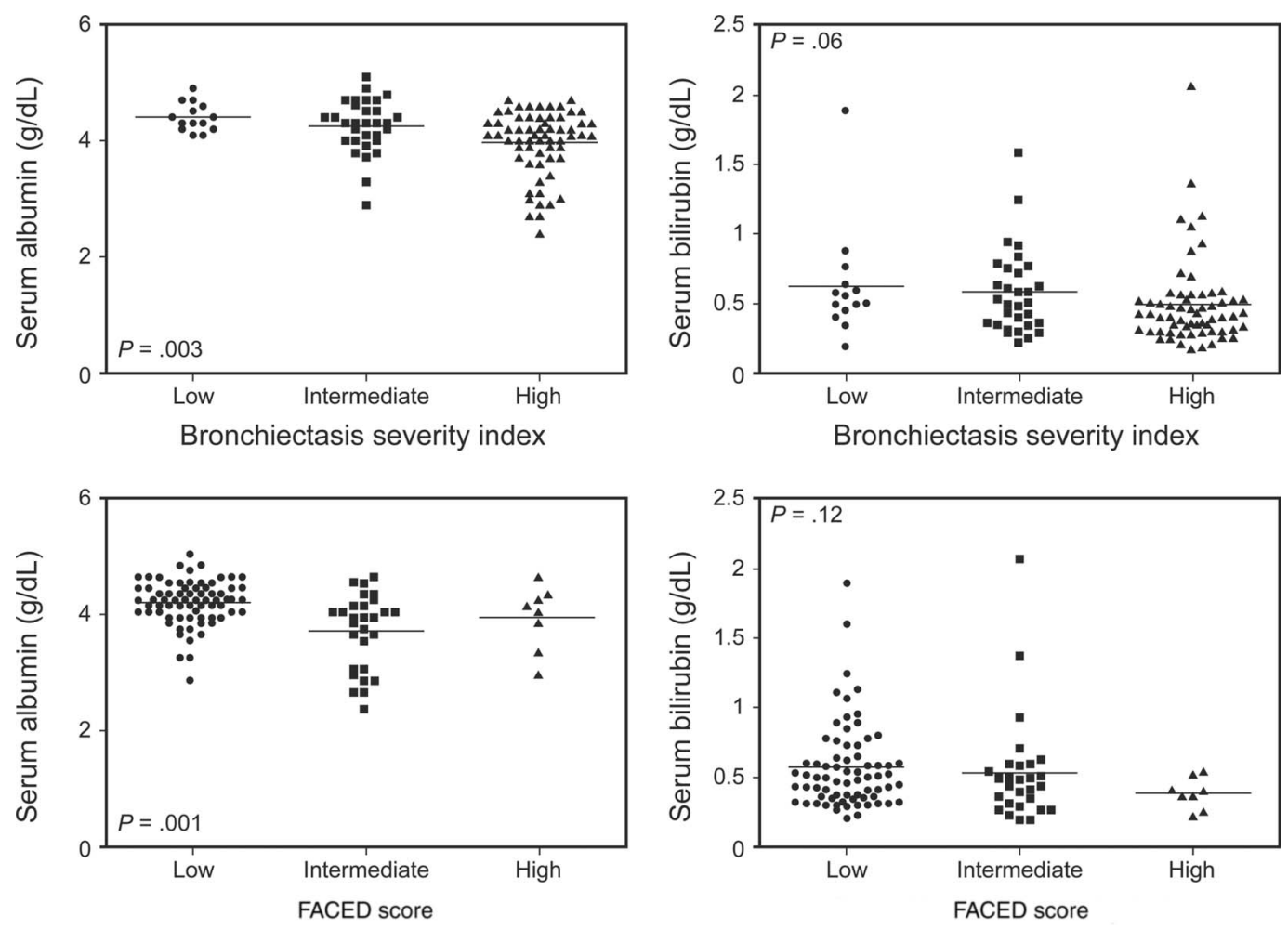

Fig. 3. Differences of serum albumin and bilirubin level according to the severity groups.

were increased in bronchiectasis subjects with bacterial colonization. Although the current study did not aim to compare the inflammatory markers between bronchiectasis subjects and control subjects, our results showed that systemic inflammatory biomarkers, including the C-reactive protein level and platelet/lymphocyte ratio, were positively correlated with the severity of non-CF bronchiectasis.

Serum bilirubin and uric acid levels are known to play important roles in human respiratory disease through antioxidant activity. A high level of serum uric acid was associated with a decreased incidence of COPD and lung cancer, ${ }^{32}$ and the uric acid level was negatively associated with the severity of COPD. ${ }^{33}$ Horsfall et al ${ }^{34}$ reported that a high level of serum bilirubin was associated with a low risk of COPD and lung cancer, similar to uric acid. A significant association between the serum bilirubin level and disease severity and progression of COPD was also proven by Apperley et al. ${ }^{35}$ The current study revealed that the level of serum bilirubin was negatively correlated with the BSI. Furthermore, the serum bilirubin level was independently and significantly associated with the BSI in multivariate linear regression analysis. The level of serum uric acid showed a negative correlation and was independently 
Table 5. Multiple Linear Regression Analysis of the Laboratory Variables in Male Subjects With Non-Cystic Fibrosis Bronchiectasis

\begin{tabular}{|c|c|c|c|c|}
\hline Dependent Variables & Explanatory Variables & $\beta$ Coefficient & $95 \% \mathrm{CI}$ & $P$ \\
\hline \multirow[t]{8}{*}{ BSI } & Neutrophil (\%) & 0.06 & -0.30 to 0.42 & .75 \\
\hline & Lymphocyte (\%) & 0.10 & -0.32 to 0.52 & .63 \\
\hline & PLR & 0.01 & -0.02 to 0.03 & .51 \\
\hline & Hemoglobin (g/dL) & 0.39 & -0.29 to 1.06 & .26 \\
\hline & Creatinine (mg/dL) & 1.12 & -1.68 to 3.92 & .42 \\
\hline & Uric acid (mg/dL) & -0.66 & -1.30 to -0.01 & .045 \\
\hline & Albumin (g/dL) & -4.36 & -6.33 to -2.40 & $<.001$ \\
\hline & Bilirubin (mg/dL) & -3.00 & -5.61 to -0.39 & .03 \\
\hline \multirow[t]{8}{*}{ FACED score } & Neutrophil (\%) & -0.10 & -0.24 to 0.05 & .19 \\
\hline & Lymphocyte (\%) & -0.17 & -0.35 to 0.01 & .07 \\
\hline & NLR & -0.25 & -0.65 to 0.15 & .22 \\
\hline & PLR & -0.00 & -0.01 to 0.01 & .73 \\
\hline & Hemoglobin (g/dL) & 0.08 & -0.21 to 0.37 & .59 \\
\hline & Creatinine (mg/dL) & -0.65 & -1.71 to 0.31 & .18 \\
\hline & Albumin (g/dL) & -1.58 & -2.41 to -0.75 & $<.001$ \\
\hline & Bilirubin (mg/dL) & -0.80 & -1.85 to 0.25 & .13 \\
\hline
\end{tabular}

$\overline{\mathrm{BSI}}=$ bronchiectasis severity index

PLR $=$ platelet/lymphocyte ratio

$\mathrm{NLR}=$ neutrophl/lymphocyte ratio

associated with BSI in male subjects with non-CF bronchiectasis in this study. These results indicate that interactions between oxidative stress and antioxidant activity are vital in the progression of non-CF bronchiectasis.

Not only the nutritional status, but also the inflammatory response, is responsible for hypoalbuminemia. ${ }^{36}$ Chronic respiratory diseases increase energy expenditure because of an elevated work load with respect to breathing. Simultaneously, chronic systemic and airway inflammation leads to hypoalbuminemia via the elevated transfer of albumin to the extravascular compartment. Hypoalbuminemia is associated with increased morbidity and mortality in chronic respiratory diseases, including COPD and cystic fibrosis. Malnutrition was associated with worse lung function, ${ }^{37}$ and a low level of serum albumin was a predictor of low $\mathrm{FEV}_{1}$ in subjects with cystic fibrosis. ${ }^{38}$ The prognostic importance of albumin is more evident in subjects with COPD. A low level of serum albumin was an independent predictor of a prolonged hospital stay and mortality in subjects with COPD exacerbation. ${ }^{39,40}$ Furthermore, Chen et $\mathrm{al}^{41}$ reported that hypoalbuminemia was a strong risk factor for acute respiratory failure in subjects with COPD. The level of serum albumin showed an independent negative association with both the BSI and FACED scores in this study. Although these results cannot confirm the prognostic significance of the serum albumin level, it is highly possible that serum albumin plays a role in subjects with non-CF bronchiectasis.

Some limitations of the current study need to be acknowledged. First, this study was a retrospective observa- tional analysis of previous medical records. Thus, many subjects were excluded because pulmonary function and sputum bacteriologic test data were absent, consequently resulting in the inclusion of a relatively small number of subjects. There is a chance that many subjects with milder disease were excluded because pulmonary function and sputum studies may not be performed in asymptomatic patients with non-CF bronchiectasis. Second, the causal relationship between the laboratory markers and disease severity score is unclear because the study used a crosssectional design. Although the level of serum albumin was an independent predictor of disease severity, longitudinal analysis will be needed for further confirmation. Third, this study was indirectly aiming to discover prognostic biomarkers using the BSI and FACED scores. Finally, the etiology of non-CF bronchiectasis could not be verified. The etiologies of non-CF bronchiectasis are diverse, and different clinical features may exist according to phenotype. Nevertheless, this is the first study that verified the clinical usefulness of serum albumin in subjects with non-CF bronchiectasis based on the BSI and FACED scores.

\section{Conclusions}

Among numerous laboratory variables, including the serum albumin level, bilirubin level, hemoglobin level, C-reactive protein level, platelet/lymphocyte ratio, and uric acid level, all of which showed a significant correlation with the BSI and/or FACED score, the level of serum 


\section{Serum Albumin in Bronchiectasis}

albumin was the marker most strongly (and independently) associated with non-CF bronchiectasis severity. Thus, the serum albumin level may serve as a simple and relevant prognostic biomarker in subjects with non-CF bronchiectasis.

\section{REFERENCES}

1. Bilton D. Update on non-cystic fibrosis bronchiectasis. Curr Opin Pulm Med 2008;14(6):595-599.

2. De Soyza A, Brown JS, Loebinger MR. Research priorities in bronchiectasis. Thorax 2013;68(7):695-696.

3. Pasteur MC, Bilton D, Hill AT. British Thoracic Society guideline for non-CF bronchiectasis. Thorax 2010;65(Suppl 1):i1-i58.

4. Martínez-García MÁ, de Gracia J, Vendrell Relat M, Girón RM, Máiz Carro L, de la Rosa Carrillo D, Olveira C. Multidimensional approach to non-cystic fibrosis bronchiectasis: the FACED score. Eur Respir J 2014;43(5):1357-1367.

5. Chalmers JD, Goeminne P, Aliberti S, McDonnell MJ, Lonni S, Davidson J, et al. The bronchiectasis severity index: an international derivation and validation study. Am J Respir Crit Care Med 2014; 189(5):576-585.

6. Leung TF, Ko FW, Wong GW. Recent advances in asthma biomarker research: therapeutic advances in respiratory disease 2013; 7(5):297-308

7. Sin DD, Hollander Z, DeMarco ML, McManus BM, Ng RT. Biomarker development for chronic obstructive pulmonary disease: from discovery to clinical implementation. Am J Respir Crit Care Med 2015;192(10):1162-1170

8. Petsky HL, Kynaston JA, Turner C, Li AM, Cates CJ, Lasserson TJ, et al. Tailored interventions based on sputum eosinophils versus clinical symptoms for asthma in children and adults. Cochrane Database Syst Rev 2007;(2):CD005603.

9. Smith AD, Cowan JO, Brassett KP, Filsell S, McLachlan C, MontiSheehan G, et al. Exhaled nitric oxide: a predictor of steroid response. Am J Respir Crit Care Med 2005;172(4):453-459.

10. Leung JM, Sin DD. Biomarkers in airway diseases. Can Respir J 2013;20(3):180-182.

11. Nobakht M Gh BF, Aliannejad R, Rezaei-Tavirani M, Taheri S, Oskouie AA. The metabolomics of airway diseases, including COPD, asthma and cystic fibrosis. Biomarkers 2015;20(1):5-16.

12. Rossi R, De Palma A, Benazzi L, Riccio AM, Canonica GW, Mauri P. Biomarker discovery in asthma and COPD by proteomic approaches. Proteomics Clin Appl 2014;8(11):901-915.

13. Hansell DM, Bankier AA, MacMahon H, McLoud TC, Müller NL, Remy J. Fleischner Society: glossary of terms for thoracic imaging. Radiology 2008;246(3):697-722.

14. Griffith DE, Aksamit T, Brown-Elliott BA, Catanzaro A, Daley C, Gordin F, et al. An official ATS/IDSA statement: diagnosis, treatment, and prevention of nontuberculous mycobacterial diseases. Am J Respir Crit Care Med 2007;175(4):367-416.

15. Miller MR, Hankinson J, Brusasco V, Burgos F, Casaburi R, Coates A, et al. Standardisation of spirometry. Eur Respir J 2005;26(2):319338.

16. Reiff DB, Wells AU, Carr DH, Cole PJ, Hansell DM. CT findings in bronchiectasis: limited value in distinguishing between idiopathic and specific types. AJR Am J Roentgenol 1995;165(2): 261-267.

17. Brill SE, Patel AR, Singh R, Mackay AJ, Brown JS, Hurst JR. Lung function, symptoms and inflammation during exacerbations of non- cystic fibrosis bronchiectasis: a prospective observational cohort study. Respir Res 2015;16:16.

18. Onen ZP, Gulbay BE, Sen E, Yildiz OA, Saryal S, Acican T, Karabiyikoglu G. Analysis of the factors related to mortality in patients with bronchiectasis. Respir Med 2007;101(7):1390-1397.

19. King PT, Holdsworth SR, Freezer NJ, Villanueva E, Holmes PW. Characterisation of the onset and presenting clinical features of adult bronchiectasis. Respir Med 2006;100(12):2183-2189.

20. Morrissey BM, Harper RW. Bronchiectasis: sex and gender considerations. Clin Chest Med 2004;25(2):361-372.

21. Loebinger MR, Wells AU, Hansell DM, Chinyanganya N, Devaraj A, Meister M, Wilson R. Mortality in bronchiectasis: a long-term study assessing the factors influencing survival. Eur Respir J 2009; 34(4):843-849.

22. King P, Holdsworth S, Freezer N, Holmes P. Bronchiectasis. Int Med J 2006;36(11):729-737.

23. Hurst JR, Elborn JS, De Soyza A. COPD-bronchiectasis overlap syndrome. Eur Respir J 2015;45(2):310-313.

24. Martínez-García MA, de la Rosa Carrillo D, Soler-Cataluña JJ, Donat-Sanz Y, Serra PC, Lerma MA, et al. Prognostic value of bronchiectasis in patients with moderate-to-severe chronic obstructive pulmonary disease. Am J Respir Crit Care Med 2013;187(8):823831.

25. Goeminne PC, Nawrot TS, Ruttens D, Seys S, Dupont LJ. Mortality in non-cystic fibrosis bronchiectasis: a prospective cohort analysis. Respir Med 2014;108(2):287-296.

26. Minov J, Karadzinska-Bislimovska J, Vasilevska K, Stoleski S, Mijakoski D. Assessment of the non-cystic fibrosis bronchiectasis severity: the FACED score vs the bronchiectasis severity index. Open Respir Med J 2015;9:46-51.

27. McDonnell MJ, Aliberti S, Goeminne PC, Dimakou K, Zucchetti SC, Davidson J, et al. Multidimensional severity assessment in bronchiectasis: an analysis of seven European cohorts. Thorax 2016; 71(12):1110-1118

28. Thomsen M, Ingebrigtsen TS, Marott JL, Dahl M, Lange P, Vestbo $\mathrm{J}$, Nordestgaard BG. Inflammatory biomarkers and exacerbations in chronic obstructive pulmonary disease. JAMA 2013;309(22):23532361.

29. Martínez-García MA, Perpiñá-Tordera M, Román-Sánchez P, SolerCataluña JJ, Carratalá A, Yago M, Pastor MJ. [The association between bronchiectasis, systemic inflammation, and tumor necrosis factor alpha]. Arch Bronconeumol 2008;44(1):8-14.

30. Gale NS, Bolton CE, Duckers JM, Enright S, Cockcroft JR, Shale DJ. Systemic comorbidities in bronchiectasis. Chron Respir Dis 2012; 9(4):231-238

31. Ergan Arsava B, Cöplü L. Does airway colonization cause systemic inflammation in bronchiectasis? Tuberkuloz ve toraks 2011;59(4): 340-347.

32. Horsfall LJ, Nazareth I, Petersen I. Serum uric acid and the risk of respiratory disease: a population-based cohort study. Thorax 2014; 69(11):1021-1026.

33. Nicks ME, O'Brien MM, Bowler RP. Plasma antioxidants are associated with impaired lung function and COPD exacerbations in smokers. COPD 2011;8(4):264-269.

34. Horsfall LJ, Rait G, Walters K, Swallow DM, Pereira SP, Nazareth I, Petersen I. Serum bilirubin and risk of respiratory disease and death. JAMA 2011;305(7):691-697.

35. Apperley S, Park HY, Holmes DT, Man SF, Tashkin D, Wise RA, et al. Serum bilirubin and disease progression in mild COPD. Chest 2015;148(1):169-175.

36. Don BR, Kaysen G. Serum albumin: relationship to inflammation and nutrition. Semin Dial 2004;17(6):432-437.

37. Steinkamp G, Wiedemann B. Relationship between nutritional status and lung function in cystic fibrosis: cross sectional and longitudinal 


\section{Serum Albumin in Bronchiectasis}

analyses from the German CF quality assurance (CFQA) project. Thorax 2002;57(7):596-601.

38. Souza dos Santos Simon MI, Drehmer M, de Abreu E Silva FA, Hoffmann A, Druck Ricachinewsky C, de Fonseca Andrade Procianoy E, et al. Association of nutritional status, plasma, albumin levels and pulmonary function in cystic fibrosis. Nutr Hosp 2011; 26(6):1322-1327.

39. Wang Y, Stavem K, Dahl FA, Humerfelt S, Haugen T. Factors associated with a prolonged length of stay after acute exacerbation of chronic obstructive pulmonary disease (AECOPD). Int J Chron Obstruct Pulmon Dis 2014;9:99-105.

40. Gunen H, Hacievliyagil SS, Kosar F, Mutlu LC, Gulbas G, Pehlivan E, et al. Factors affecting survival of hospitalised patients with COPD. Eur Respir J 2005;26(2):234-241.

41. Chen CW, Chen YY, Lu CL, Chen SC, Chen YJ, Lin MS, Chen W. Severe hypoalbuminemia is a strong independent risk factor for acute respiratory failure in COPD: a nationwide cohort study. Int J Chron Obstruct Pulmon Dis 2015;10:1147-1154. 